\title{
Parâmetros Populacionais do Rebanho Sindi Registrado no Brasil
}

\author{
Fábio José Carvalho Faria1, Anibal Eugênio Vercesi Filho', Fernando Enrique Madalena², \\ Luiz Antônio Josahkian ${ }^{3}$
}

\begin{abstract}
RESUMO - O objetivo deste trabalho foi descrever a estrutura da população do rebanho Sindi registrado no Brasil. Foram geradas estatísticas descritivas da distribuição do número de progênies, estimados o intervalo de gerações e o tamanho efetivo da população, usando o registro genealógico de animais nascidos entre 1955-1998. O intervalo de gerações médio calculado para o arquivo completo foi de 7,65 anos e para o período 1994-1998 foi de 7,96 anos. Os tamanhos efetivos da população nos quatro períodos (1979-83, 1984-88, 198993 e 1994-98) foram, respectivamente, 501, 26, 20 e 19.
\end{abstract}

Palavras-chave: estrutura populacional, intervalo de gerações, tamanho efetivo, variância de progênie

\section{Population Parameters of the Registered Red Sindi in Brazil}

\begin{abstract}
The aim of this study was to describe the population structure of the registered Red Sindhi cattle in Brazil. Descriptive statistics of the distribution of the number of progeny are presented, and the generation interval and effective population size were estimated, using pedigree records of animals registered from 1955 to 1998 . The observed generation interval for the whole time period was $7.65 \mathrm{yr}$, and for the period 1994-1998 it was $7.96 \mathrm{yr}$. The effective population size for the following periods (1979-83, 1984-88, 1989-93 and 1994-98) were, respectively, 501, 26, 20 and 19.
\end{abstract}

Key Words: population structure, generation interval, effective size, progeny variance

\section{Introdução}

A raça Sindi é classificada por JOSHI e PHILIPS (1954) como pertencente ao terceiro grupo das raças indianas, onde o principal representante é o gado Gir. É originária do Paquistão, numa região de clima semi-árido com precipitação anual média entre $250-300 \mathrm{~mm}^{3}$. Os animais desta raça são em geral pequenos, com cabeças pequenas de perfis subconvexos, chifres grossos e curtos, orelhas de tamanho médio e largas, e pelagens vermelha, vermelha escura, retinta ou castanha.

Acredita-se que o primeiro exemplar da raça a chegar ao Brasil foi um reprodutor que veio para o estado da Bahia, em 1850 (SANTIAGO, 1986). O rebanho Sindi brasileiro está localizado principalmente nas regiões Norte e Nordeste. Em função de ser uma das principais raças zebuínas leiteiras e de sua reputação em rusticidade (MADALENA, 2001), o Sindi constitui importante recurso genético a ser explorado no Brasil e em outros países tropicais.

De acordo com a Food and Agriculture Organization of the United Nations (FAO), os animais domésticos produzem menos de $30 \%$ das necessidades humanas de alimentação e da agricultura, sob a forma de leite e derivados, ovos, carne, fibra, fertilizantes para cultivo e tração. Esta contribuição é obtida a partir de aproximadamente 4.500 raças provenientes de 40 espécies, desenvolvidas ao longo dos últimos 12.000 anos, que representa o patrimônio genético disponível, o qual deverá satisfazer as demandas futuras. A cada mês estão desaparecendo seis raças e, de acordo com os últimos levantamentos, $30 \%$ das raças mundiais estão em perigo de extinção (DOMESTIC..., 1998).

O objetivo deste trabalho foi descrever a composição, tamanho efetivo e o intervalo de gerações do gado Sindi no Brasil, parâmetros estes de interesse para o melhoramento genético e a conservação da raça.

\footnotetext{
1 Aluno de Doutorado em Ciência Animal, Departamento de Zootecnia, Escola de Veterinária da UFMG, Av. Antônio Carlos 6627, Cx. P. 567, CEP 30123-970, Belo Horizonte - MG. E.mail: fariafjc@dedalus.lcc.ufmg.br

2 Professor do Departamento de Zootecnia, Escola de Veterinária da UFMG, Av. Antônio Carlos 6627, Cx. P. 567, CEP 30123-970, Belo Horizonte - MG. E.mail: fermadal@dedalus.lcc.ufmg.br

3 Superintendente técnico da Associação Brasileira de Criadores de Zebu, Praça Vicente Rodrigues da Cunha, 110 BI 1, CEP 38022-330, Uberaba - MG. Professor de Melhoramento Animal da Faculdade de Agronomia e Zootecnia de Uberaba - FAZU.
} 


\section{Material e Métodos}

Foram utilizados os dados do registro genealógico da raça Sindi, disponíveis sob processamento eletrônico, da Associação Brasileira de Criadores de Zebu (ABCZ). O arquivo de dados consistiu de 4.884 observações entre os anos de 1955 a 1998, referentes à amostragem de 8.080 registros (www.abcz.org.br). As informações referentes a cada animal consistiam de: pai, mãe, sexo, categoria de registro $(\mathrm{PO}=$ puro de origem; LA = livro aberto), dia, mês e ano de nascimento, criador e proprietário.

Inicialmente foram geradas estatísticas descritivas desta população. O intervalo médio de gerações $(\bar{L})$ foi calculado de acordo a seguinte fórmula:

$$
L=1 / 4 *\left(L_{P M}+L_{P F}+L_{M M}+L_{M F}\right)
$$

em que: $\mathrm{L}_{\mathrm{PM}}=$ intervalo médio entre pais e filhos; $\mathrm{L}_{\mathrm{PF}}=$ intervalo médio entre pais e filhas; $\mathrm{L}_{\mathrm{MM}}=$ intervalo médio entre mães e filhos; $\mathrm{L}_{\mathrm{MF}}=$ intervalo médio entre mães e filhas.

Estimou-se também o intervalo médio de gerações dos pais e avós do último período como: pai de pai, pai de mãe, mãe de mãe e mãe de pai. O banco de dados foi separado em quatro períodos, compreendendo os anos 1979-83, 1984-88, 1989-93 e 1994-98, em razão do maior número e consistência dos arquivos. Em cada período foram calculados o número de machos e fêmeas em reprodução, a média, variância e covariância do número de progênie (filhos e filhas por reprodutor/matriz) e o intervalo de gerações médio. O tamanho efetivo da população $(\mathrm{Ne})$ foi calculado para cada período, segundo fórmula proposta por HILL (1972):

$$
\begin{gathered}
\frac{1}{N e}=\frac{1}{16 M \bar{L}}\left[2+\sigma_{\mathrm{mm}}^{2}+2\left(\frac{M}{F}\right) \operatorname{cov}(\mathrm{mm}, \mathrm{mf})+\left(\frac{M}{F}\right)^{2} \sigma_{\mathrm{mf}}^{2}\right]+\frac{1}{16 F \bar{L}}[2+ \\
\left.\left(\frac{F}{M}\right)^{2} \sigma_{\mathrm{fm}}^{2}+2\left(\frac{F}{M}\right) \operatorname{cov}(\mathrm{fm}, \mathrm{ff})+\sigma_{\mathrm{ff}}^{2}\right]
\end{gathered}
$$

em que: $\mathrm{M}=$ número de pais no período; $\mathrm{F}=$ número de mães no período; $\sigma^{2}{ }_{\mathrm{mm}}=$ variância do número de filhos por pai; $\sigma^{2}{ }_{\mathrm{mf}}=$ variância do número de filhas por pai; $\sigma_{\text {fm }}^{2}=$ variância do número de filhos por mãe; $\sigma_{\mathrm{ff}}^{2}=$ variância do número de filhas por mãe; $\operatorname{cov}(\mathrm{mm}, \mathrm{mf})=$ covariância do número de filhos de ambos os sexos por pai; cov $(\mathrm{fm}, \mathrm{ff})=$ covariância do número de filhos de ambos os sexos por mãe.

\section{Resultados e Discussão}

Na Tabela 1, constam o número de criadores e o número de animais registrados, separados por sexo,
Tabela 1 - Distribuição de criadores de acordo com o número de animais registrados separados por sexo de 1989 a 1998

Table 1 - Distribution of breeders according to number of registered animals and sex from 1989 to 1998

\begin{tabular}{ccc}
\hline Machos & Fêmeas & Ambos os sexos \\
Males & Females & Both sexes \\
\hline
\end{tabular}

\begin{tabular}{lccc}
$\begin{array}{l}\text { Número de animais } \\
\text { Number of animals }\end{array}$ & \multicolumn{3}{c}{$\begin{array}{c}\text { Número de criadores } \\
\text { Number of breeders }\end{array}$} \\
\hline $1-25$ & 6 & 6 & 7 \\
$26-50$ & 1 & 3 & 1 \\
$51-75$ & 1 & - & 2 \\
$76-100$ & - & - & - \\
$101-200$ & 1 & 1 & 1 \\
$\geq 201$ & 1 & 2 & 2 \\
Total & 10 & 12 & 13 \\
\hline
\end{tabular}

no período de 1989 a 1998. A escolha deste período teve o intuito de retratar o número de criadores efetivos da raça. Nota-se o reduzido número de criadores desta raça no Brasil. Além disso, existe concentração de animais com poucos criadores. Neste período, foram registrados 2260 animais, dos quais 988 machos e 1272 fêmeas. Do total de animais registrados, $60 \%$ pertenciam a um único criador. Fato que deve ser mencionado é a existência de registro seletivo, onde o número de fêmeas registradas foi sempre superior ao de machos. A estrutura atual deste grupamento permite evidenciar que a raça está conservada em poucos rebanhos, com um principal. A Figura 1 apresenta o diagrama de parentesco, passível de identificação, dos animais registrados, evidenciando que a medida que regrediu-se no arquivo de pedigree foi-se perdendo informações sobre a ascendência dos animais.

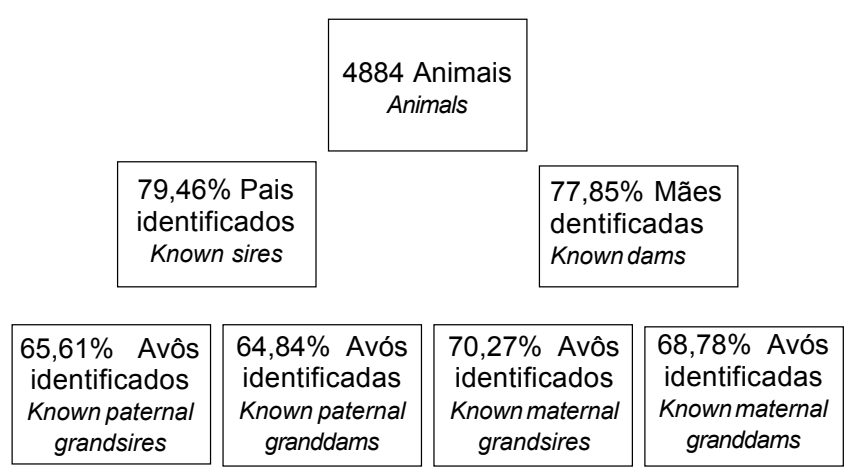

Figura 1 -Grau de identificação de ancestrais. Figure 1 - Degree of ancestors identification. 
Do total de 1489 machos registrados entre 1955 e 1998, 133 animais foram identificados como pais (reprodutores), representando uma proporção de 0,089 . O número médio de filhos e filhas por reprodutor foi 13,4 e 19,6 respectivamente, sendo a média de crias de ambos os sexos de 29,18. Dentre os reprodutores, $7 \%$ dos animais contribuíram com $29 \%$ das filhas e $5 \%$ dos animais foram pais de $31 \%$ dos machos nascidos no período estudado. A idade média do pai quando do registro da primeira cria foi de 6 anos. $\mathrm{Na}$ Tabela 2, estão expostos os números de reprodutores separados por classe de acordo com o sexo da progênie. Verificou-se que, do total de 133 reprodutores, $76 \%$ contribuíram com o registro de machos, ao passo que houve maior proporção de pais de fêmeas $96 \%$, evidenciando seleção mais intensa para o registro de machos que irão contribuir com as próximas gerações.

Foram identificadas 1489 mães entre 1955 e 1998, que representam $43 \%$ das fêmeas registradas. O número médio de filhos e filhas por mãe foi 1,82 e 1,95 respectivamente, sendo a média de crias de ambos os sexos de 2,55. Dentre as mães, $64 \%$ tiveram de uma a duas filhas e $38 \%$ tiveram de um a dois filhos durante o período estudado. A distribuição do número de filhos por mãe, separados por sexo, pode ser visualizada na Tabela 3. Do total de 1.489 mães, $49 \%$ contribuíram com o registro de machos, ao passo que $85 \%$ mães contribuíram para o registro de fêmeas, demonstrando maior seleção para mães de machos que para mães de fêmeas.

$\mathrm{Na}$ Tabela 4, estão apresentadas as estimativas dos intervalos de gerações para as quatro passagens gaméticas. Os intervalos foram obtidos após separa-

Tabela 2 - Distribuição de reprodutores de acordo com o número de filhos separados por sexo

Table 2 - Distribution of sires according to progeny number and sex

\begin{tabular}{lccc}
\hline & $\begin{array}{c}\text { Filhos } \\
\text { Sons }\end{array}$ & $\begin{array}{c}\text { Filhas } \\
\text { Daughters }\end{array}$ & $\begin{array}{c}\text { Ambos os sexos } \\
\text { Both sexes }\end{array}$ \\
\hline $\begin{array}{l}\text { Número de filhos } \\
\text { Progeny numbers }\end{array}$ & \multicolumn{3}{c}{ Numero de reprodutores } \\
Number of sires \\
\hline $1-50$ & 94 & 118 & 110 \\
$51-100$ & 6 & 7 & 15 \\
$101-150$ & 2 & 3 & 4 \\
$\geq 151$ & - & - & 4 \\
Total & 102 & 128 & 133 \\
\hline
\end{tabular}

ção do banco de dados em dois períodos. No primeiro arquivo, considerou-se todo o período de dados. Já no segundo, o intervalo de gerações foi estimado a partir dos animais nascidos entre 1994 e 1998, onde foram avaliadas as passagens de pais de pais deste período. Observa-se, nesta Tabela, um incremento do intervalo médio de gerações no último período, em função principalmente do aumento no intervalo dos passos: pai - filho e mãe - filho, o que reflete uso prolongado de certos animais na reprodução.

Esses intervalos são similares aos demais encontrados para raças zebuínas criadas no Brasil (Tabela 5). A otimização do intervalo médio de gerações é de fundamental importância em programa de melhoramento genético, pois intervalos muito grandes diminuem o ganho genético anual para as características selecionadas, o que leva a menor retorno econômico do programa. Vale salientar o reduzido intervalo de gerações descrito por RAZOOK et al. (1993), nos rebanhos da Estação Experimental de Zootecnia de Sertãozinho - SP, onde os machos iniciam a vida reprodutiva com dois anos de idade e permanecem em serviço por duas estações de monta, resultando num intervalo médio de pai para filhos de 3,66 e 3,74 anos para os rebanhos Nelore e Guzerá, respectivamente. A utilização de reprodutores por tempo limitado proporciona redução no intervalo de gerações, além da possível redução na variância do tamanho de família que tende a minimizar decréscimos no tamanho efetivo da população.

As estimativas dos tamanhos efetivos da população foram 501, 26, 20 e 19 animais para os períodos 1979-83, 1984-88, 1989-93 e 1994-98, respectivamente. O número de reprodutores (Tabela 6) foi similar nestes períodos, entretanto, a relação

Tabela 3 - Distribuição de matrizes de acordo com o número de filhos separados por sexo

Table 3 - Distribution of dams according to progeny number and sex

\begin{tabular}{|c|c|c|c|}
\hline & $\begin{array}{l}\text { Filhos } \\
\text { Sons }\end{array}$ & $\begin{array}{c}\text { Filhas } \\
\text { Daughters }\end{array}$ & $\begin{array}{c}\text { Ambos os sexos } \\
\text { Both sexes }\end{array}$ \\
\hline $\begin{array}{l}\text { Número de filhos } \\
\text { Number of progeny }\end{array}$ & \multicolumn{3}{|c|}{$\begin{array}{l}\text { Número de mães } \\
\text { Number of dams }\end{array}$} \\
\hline$\overline{1-2}$ & 574 & 946 & 970 \\
\hline $3-4$ & 128 & 261 & 283 \\
\hline $5-6$ & 30 & 44 & 145 \\
\hline $7-8$ & 5 & 7 & 67 \\
\hline$\geq 9$ & - & 1 & 24 \\
\hline Total & 737 & 1259 & 1489 \\
\hline
\end{tabular}

Rev. bras. zootec., 30(6S):1989-1994, 2001 
Tabela 4 - Intervalo de gerações das quatro passagens gaméticas e intervalo médio Table 4 - Generation interval of four gametic pathways and average interval

\begin{tabular}{|c|c|c|c|c|c|}
\hline & \multicolumn{5}{|c|}{$\begin{array}{c}\text { Passagens gaméticas } \\
\text { Gametic pathways }\end{array}$} \\
\hline & $\begin{array}{l}\text { Pai - filho } \\
\text { Sire - son }\end{array}$ & $\begin{array}{c}\text { Pai - filha } \\
\text { Sire - daughter }\end{array}$ & $\begin{array}{l}\text { Mãe - filho } \\
\text { Dam - son }\end{array}$ & $\begin{array}{c}\text { Mãe - filha } \\
\text { Dam - daughter }\end{array}$ & $\begin{array}{l}\text { Média } \\
\text { Average }\end{array}$ \\
\hline & & & . ano (year)... & .... & $\ldots \ldots \ldots \ldots$ \\
\hline 1955 a 1998 & 7,44 & 8,13 & 7,12 & 7,93 & 7,65 \\
\hline 1994 a 1998 & 9,11 & 7,47 & 8,08 & 7,21 & 7,96 \\
\hline
\end{tabular}

fêmea:macho dobrou, passando de 7,11:1 a 15,28:1 entre o primeiro e o último período, respectivamente. Ficam claros nestes períodos aumentos do número médio de filhos e da variância do número de progênie. De acordo com WRIGHT (1938), a oscilação do Ne pode ser atribuída a fatores como flutuações no tamanho da população, desvios da razão de sexos de 1:1 e diferenças no sucesso reprodutivo.

Quando poucos animais são pais de muitos descendentes ocorre aumento na variância do tamanho de família que é inversamente proporcional ao tamanho efetivo da população $\left(\sigma_{\mathrm{f}}^{2}=1 / \mathrm{Ne}\right)$. Por sua vez, o tamanho efetivo da população também é inversamente proporcional à taxa de endogamia $(\Delta \mathrm{F}=1 / 2 \mathrm{Ne})$. A evolução do tamanho efetivo e a variância do tamanho de família de pais podem ser vistas na Figura 2.

Para a raça Sindi no Brasil, o tamanho efetivo de 19 animais é inquietador. Segundo GODDARD e SMITH (1990), um Ne mínimo de 40 por geração é necessário para maximizar o retorno econômico, em população selecionada para produção de leite, o que deve ser levado em conta quando da criação de

Tabela 5 - Intervalo de gerações de diferentes raças zebuínas Table 5 - Generation interval of different zebu breeds

\begin{tabular}{lcc}
\hline $\begin{array}{l}\text { Raça } \\
\text { Breed }\end{array}$ & $\begin{array}{c}\text { Intervalo (anos) } \\
\text { Interval (years) }\end{array}$ & $\begin{array}{c}\text { Autor } \\
\text { Author }\end{array}$ \\
\hline Gir Mocho & 6,56 & FARIA et al. (2001a) \\
Gir & 8,34 & FARIA et al. (2001b) \\
Guzerá & 7,93 & FARIA et al. (2001c) \\
Indubrasil & 7,39 & FARIA et al. (2001d) \\
Nelore & 8,33 & FARIA et al. (2001e) \\
Nelore Mocho & 7,0 & FARIA et al. (2001f) \\
Tabapuã & 7,27 & FARIA et al. (2001g) \\
Gir & 8,02 & QUEIROZ LOBO $(1993)$ \\
Nelore & 7,0 & BRASIL et al. (1990) \\
Nelore & 5,05 & RAZOOK et al. (1993) \\
Guzerá & 5,12 & RAZOOK et al. (1993) \\
Sahiwal & 6,12 & HERRON(1978) \\
\hline
\end{tabular}

programas que visem ao melhoramento deste grupamento. MEUWISSEN e WOOLLIAMS (1994) recomendam valores entre 30 e 250 para prevenir decréscimos no valor adaptativo. $\mathrm{O}$ monitoramento deste parâmetro é muito importante, visto a sua influência na variação genética. Entretanto, não foram encontradas estimativas desta natureza em raças bovinas brasileiras.

A idade média das mães ao registro da primeira e da última cria foi 6,20 e 10,07 anos, respectivamente, o que refletiu em "vida útil", para fins de registro, de 3,87 anos. O intervalo médio entre registros da mesma vaca foi 1,79 anos. Ressalva-se que a idade média ao primeiro registro pode não corresponder à idade ao primeiro parto, assim como o intervalo médio entre registros pode não corresponder ao intervalo médio de partos, pelo fato de algumas crias não terem sido registradas.

Em revisão feita pelos autores, foram identificados 99 trabalhos publicados sobre a raça Sindi de 1991 a 2000 na literatura mundial. A quase totalidade apresentou resultados de cruzamentos com raças euro-

Tabela 6 - Número de reprodutores, média e variância de número de progênie para os quatro períodos

Table 6 - Number of sires, progeny number average and variance for the analyzed periods

\begin{tabular}{|c|c|c|c|}
\hline \multirow[b]{2}{*}{$\begin{array}{l}\text { Períodos } \\
\text { Periods }\end{array}$} & \multirow[b]{2}{*}{$\begin{array}{l}\text { Número de } \\
\text { reprodutores } \\
\text { Number of sires }\end{array}$} & \multicolumn{2}{|c|}{$\begin{array}{l}\text { Número de progênie } \\
\text { por reprodutor } \\
\text { Progeny number per sire }\end{array}$} \\
\hline & & $\begin{array}{l}\text { Média } \\
\text { Average }\end{array}$ & $\begin{array}{c}\text { Variância } \\
\text { Variance }\end{array}$ \\
\hline 1979 a 1983 & 43 & 11,32 & 99,74 \\
\hline 1984 a 1988 & 49 & 15,83 & 735,63 \\
\hline 1989 a 1993 & 45 & 24,26 & 754,47 \\
\hline 1994 a 1998 & 39 & 28,38 & 745,13 \\
\hline
\end{tabular}

Rev. bras. zootec., 30(6S):1989-1994, 2001 


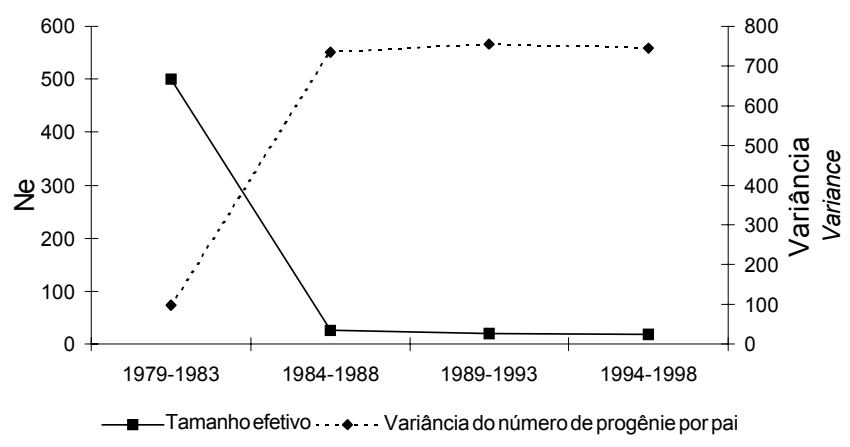

Figura 2 - Tamanho efetivo $\left(\mathrm{N}_{\mathrm{e}}\right)$ e variância do número de progênie por pai, na raça Sindi.

Figure 2 - Effective size $\left(N_{e}\right)$ and progeny number variance per sire in the Sindi breed.

péias especializadas para produção de leite, indicando que são poucos os trabalhos descritos com o Sindi como grupamento puro.

No Paquistão, a população era composta por 1.409 .308 animais, sendo 508.455 e 29.468 fêmeas e machos em reprodução, respectivamente, em que $92 \%$ destas fêmeas eram acasaladas com machos da mesma raça. Neste país, a raça não corre perigo de extinção e apresenta crescimento, segundo dados coletados em 1986 (DOMESTIC..., 1998). Na Índia, a sua população era composta por 2.400 animais, sendo 1.350 fêmeas e 140 machos em reprodução, dos quais $85 \%$ das fêmeas eram acasaladas com reprodutores da mesma raça. Neste país a raça também não apresenta risco de extinção, apesar do número de animais estar em declínio, conforme dados coletados em 1987 (DOMESTIC..., 1998).

Levando-se em conta o número médio de crias registradas de 2,55 e "vida útil" de registro de 3,87 anos, foi estimado que uma vaca produziria em média 0,4 bezerros registrados/ano $([2,55-1] / 3,87)$. Segundo a ABCZ (2001), entre 1995 e 2000, 2.026 animais obtiveram registro de nascimento (http://www.abcz.org.br), resultando numa média de 338 registros/ano. Com a estimativa de $0,4 \mathrm{cria} /$ ano/vaca estimou-se uma população média de 845 $(338 / 0,4)$ vacas registradas em reprodução no Brasil, que formariam os estratos núcleo e multiplicador. Com base em critérios descritos pela FAO (1994), o rebanho Sindi registrado no Brasil encontra-se na categoria de populações críticas (entre 100 e 1.000 vacas), com necessidade imediata de ações que previnam a sua extinção.

Fator de preocupação para a raça no Brasil é a ausência da comercialização de sêmen, sendo que no último ano não foram realizadas colheitas de sêmen em reprodutores Sindi em várias centrais pesquisadas, e conforme a Associação Brasileira de Inseminação Artificial (ASBIA, 2001) foram vendidas apenas 965 doses de sêmen da raça em 1994, o que representou $0,03 \%$ das doses vendidas para rebanhos leiteiros no Brasil, sendo este o último ano de vendas registradas.

A "conservação comercial" de raças, independente da necessidade de medidas específicas para preservá-las, pode ser conseguida por meio de promoções com base em programas de melhoramento, avaliação e cruzamentos (MADALENA, 1992). Entretanto, dado o reduzido tamanho efetivo do rebanho Sindi registrado, a adoção de medidas que visem a aumentá-lo, tais como pesquisas com a raça, promoção e divulgação, bem como importações de animais ou materiais genéticos, torna-se necessária para tirar a raça do risco de extinção no Brasil.

\section{Conclusões}

O rebanho Sindi registrado apresenta sério risco de desaparecimento no Brasil, dado seu pequeno tamanho efetivo e a manutenção em poucos rebanhos, fato que sugere a necessidade de programas que visem a sua expansão.

\section{Agradecimento}

À ABCZ pela concessão dos dados para este trabalho, realizado com apoio do CNPq, CAPES e FAPEMIG.

\section{Referências Bibliográficas}

ABCZ. 2001. "Estatísticas", http://www.abcz.org.br 10/04/2001. ASBIA. 2001. "Evolução da Inseminação Artificial no Brasil de 1979-1999”, http://www.asbia.org.br 20/01/2001.

BRASIL, L.H.A., PEREIRA, J.C.C., PEREIRA, C.S. et al. 1990. Intervalo entre gerações e tendência genética do peso aos 365 dias de idade em um rebanho da raça Nelore. Arq. Bras. Med. Vet. Zootec., 42(1):57-66.

DOMESTIC ANIMAL DIVERSITY INFORMATION SYSTEM [s.1.]: FAO, 1998. (CD-Rom).

FAO. 1994. The management of global animal genetic resources. [Rome, Italy]: FAO. 309p.

FARIA, F.J.C., VERCESI FILHO, A.E., MADALENA, F.E. et al. Intervalo de gerações e tamanho efetivo da população da raça Gir Mocho. In: REUNIÃO ANUAL DA SOCIEDADE BRASILEIRA DE ZOOTECNIA, 38., 2001, Piracicaba. Anais... Piracicaba: SBZ, 2001a. p.482-483.

FARIA, F.J.C., VERCESI FILHO, A.E., MADALENA, F.E. et al. Intervalo de gerações e tamanho efetivo da população da

Rev. bras. zootec., 30(6S):1989-1994, 2001 
raça Gir. In: REUNIÃO ANUAL DA SOCIEDADE BRASILEIRA DE ZOOTECNIA, 38., 2001, Piracicaba. Anais... Piracicaba: SBZ, 2001b. p.484-485.

FARIA, F.J.C., VERCESI FILHO, A.E., MADALENA, F.E. et al. Intervalo de gerações e tamanho efetivo da população da raça Guzerá. In: REUNIÃO ANUAL DA SOCIEDADE BRASILEIRA DE ZOOTECNIA, 38., 2001c. Piracicaba. Anais... Piracicaba: SBZ, 2001c. p.480-481.

FARIA, F.J.C., VERCESI FILHO, A.E., MADALENA, F. E. et al. Intervalo de gerações e tamanho efetivo da população da raça Indubrasil. In: REUNIÃO ANUAL DA SOCIEDADE BRASILEIRA DE ZOOTECNIA, 38., 2001d. Piracicaba. Anais... Piracicaba: SBZ, 2001d. p.485-486.

FARIA, F.J.C., VERCESI FILHO, A.E., MADALENA, F.E. et al. Intervalo de gerações e tamanho efetivo da população da raça Nelore. In: REUNIÃO ANUAL DA SOCIEDADE BRASILEIRA DE ZOOTECNIA, 38., 2001e. Piracicaba. Anais... Piracicaba: SBZ, 2001e. p.486-488.

FARIA, F.J.C., VERCESI FILHO, A.E., MADALENA, F.E. et al. Intervalo de gerações e tamanho efetivo da população da raça Nelore Mocho. In: REUNIÃO ANUAL DA SOCIEDADE BRASILEIRA DE ZOOTECNIA, 38., 2001f. Piracicaba. Anais... Piracicaba: SBZ, 2001f. p.483-484.

FARIA, F.J.C., VERCESI FILHO, A.E., MADALENA, F.E. et al. Intervalo de gerações e tamanho efetivo da população da raça Tabapuã. In: REUNIÃO ANUAL DA SOCIEDADE BRASILEIRA DE ZOOTECNIA, 38, 2001g. Piracicaba. Anais... Piracicaba: SBZ, 2001g. p.488-489.

GODDARD, M. G., SMITH, C. 1990. Optimum number of bull sires in dairy cattle breeding. J. Dairy Sci., 73:1113-1122.

HERRON, N.D. 1978. The breed structure and genetic analysis of the pedigree Sahiwal breed in Australia. Aust. J. Agric. Res., 29:381-93.
HILL, W.G. 1972. Effective size of populations with overlapping generations. Theor. Popul. Biol., 3(3):278-288.

JOSHI, N.R., PHILIPS, R.W. 1954. El ganado cebu de la India $y$ del Pakistan. Roma: Food and Agriculture Organization. $256 \mathrm{p}$.

MADALENA, F.E. 1992. The conservation of animal genetic resources in the developing countries: a practical way forward. FAO Anim. Health. Prod. Bull., 107:77-84.

MADALENA, F.E. 2001. Bos indicus breeds and Bos indicus/ Bos taurus crosses. In: Encyclopedia of Dairy Sciences, Academic Press (no prelo).

MEUWISSEN, T.H.E., WOOLLIAMS, J. 1994. Effective sizes of livestock populations to prevent a decline in fitness. Theor. Appl. Genet., 89:1019-1026.

QUEIROZ, S.A., LÔBO, R.B. 1993. Genetic relationship, inbreeding and generation interval in registered Gir cattle in Brazil. J. Anim. Breed. Genet., 110(3):228-233.

RAZOOK, A.G., FIGUEIREDO, L.A., BONILHA NETO, L.M. et al. 1993. Intensidades de seleção e repostas diretas e correlacionadas em 10 anos de progênies de bovinos das raças Nelore e Guzerá selecionadas para peso pós desmame. Bol. Ind. Anim., 50(2):147-163.

SANTIAGO, A.A. 1986. O Zebu na Índia, no Brasil e no mundo. Campinas: Instituto Campineiro de Ensino Agrícola. 745p.

WRIGHT, S. 1938. Size of population and breeding structure in relation to evolution. Science, 87:430-431.

Recebido em: 04/05/01

Aceito em: 02/08/01 